\title{
Dosimetric study of RapidArc plans and conventional intensity modulated radiotherapy for prostate cancer involving seminal vesicles and pelvis lymph nodes
}

\author{
Birendra Kumar Rout1,2, Mukka Chandra Shekar², Alok Kumar³, Kanaparthy Raja Muralidhar4 \\ ${ }_{1}^{1}$ Department of Radiation Physics, Indo-American Cancer Institute and Research Center, Hyderabad, India \\ 2Department of Physics, Jawaharlal Nehru Technological University, Hyderabad, India \\ ${ }^{3}$ Department of Radiation Physics, Mahavir Cancer Sansthan, Patna, India \\ ${ }^{4}$ Department of Radiation Physics, American Oncology Institute, Hyderabad, India
}

Received September 23, 2015; Revised January 12, 2016; Accepted January 17, 2016; Published Online January 24, 2016

\section{Original Article}

\begin{abstract}
Purpose: The main purpose of this study is to (1) identify the continual diversity between conventional fixed field intensity modulation radiotherapy (IMRT) and RapidArc (RA) for high-risk prostate cancer; and (2) determine potential benefits and drawbacks of using for this type of treatment. Methods: A cohort of 20 prostate cases including prostate, seminal vesicles and pelvic lymph nodes was selected for this study. The primary planning target volume (PTVP) and boost planning target volume ( $\mathrm{PTV}_{\mathrm{B}}$ ) were contoured. The total prescription dose was 75.6 Gy (45 Gy to PTV $\mathrm{P}$ and an additional $21.6 \mathrm{~Gy}$ to $\mathrm{PTV}_{\mathrm{B}}$ ). Two plans were generated for each PTV: multiple 7-fields for IMRT and two arcs for RA. Results: A Sigma index (IMRT: $2.75 \pm 0.581$; RA: $2.8 \pm 0.738$ ) for PTV and (IMRT: $2.0 \pm 0.484$; RA: $2.1 \pm 0.464$ ) for PTV $_{B}$ indicated similar dose homogeneity inside the PTV. Conformity index (IMRT: $0.96 \pm 0.047$; RA: $0.95 \pm 0.059$ ) for PTVP and (IMRT: 0.97 \pm 0.015 ; RA: $0.96 \pm 0.014$ ) for PTV $_{B}$ was comparable for both the techniques. IMRT offered lower mean dose to organ at risks (OARs) compared to RA plans. Normal tissue integral dose in IMRT plan resulted $0.87 \%$ lower than RA plans. All the plans displayed significant increase (2.50 times for $\mathrm{PTV}_{\mathrm{P}}$ and 1.72 for $\mathrm{PTB}_{\mathrm{B}}$ ) in the average number of necessary monitor units (MUs) with IMRT beam. Treatment delivery time of RA was $2-6$ minutes shorter than IMRT treatment. Conclusion: For PTV including pelvic lymph nodes, seminal vesicles and prostate, IMRT offered a greater degree of OARs sparing. For PTV including seminal vesicles and prostate, RA with two arcs provided comparable plan with IMRT. RA also improved the treatment efficiency due to smaller number of MUs required.
\end{abstract}

Keywords: IMRT, RapidArc, Sigma-Index, Conformity Index, Normal Tissue Integral Dose

\section{Introduction}

Cancer is the major cause of leading deaths in the $21^{\text {st }}$ century in world with 14.1 million cases and 8.2 million deaths in $2012 .{ }^{1}$ Among them, prostate cancer stands as important due to risk of secondary malignancies associated with intensity modulation radiation therapy (IMRT) with conventional 3-dimentional conformal radiotherapy (3D-CRT). ${ }^{2}$ Among the different technologies adopted to cure the prostate cancer, external radiotherapy is recognized as one of the important treatment options. ${ }^{3-4}$ The technology aims to destroy cancer cells by minimal damaging (due to risk of secondary malignancies) to the surrounding normal tissues. It creates the best possible balance between maximizing dose to prostate cancer cells and minimization of side effects. IMRT technology for prostate tumor/cancer allows less toxicity in comparison to 3D-CRT. ${ }^{5}$ The development of IMRT technique has enabled the delivery of highly conformal

Corresponding author: Birendra Kumar Rout; Department of Radiation Physics, Indo-American Cancer Institute and Research Center, Hyderabad, India.

Cite this article as: Rout BK, Shekar MC, Kumar A, Muralidhar KR. Dosimetric Study of RapidArc plans and conventional intensity modulated radiotherapy for prostate cancer involving seminal vesicles and pelvis lymph nodes. Int J Cancer Ther Oncol 2016; 4(1):418. DOI: 10.14319/ijcto.41.8 
dose distribution to the target while limiting radiation damage to the critical organs within the tolerance limit. For the prostate cancer treatment, IMRT has been an ideal technique with the geometric relationship of the target volume to bladder, rectum and surrounding normal tissue.

RapidArc (Varian Medical Systems, Palo Alto, California, USA) is a volumetric radiotherapy technology that delivers a fast and precise sculpted 3D dose distribution with a single 360-degree rotation of the linear accelerator gantry to improve the standard of care and treatments. ${ }^{6}$ It is made possible by a treatment planning algorithm that simultaneously changes 3-parameters during treatment by tuning rotation speed of the gantry; shape of the treatment aperture using the movement of multileaf collimator leaves and delivery dose rate. Volumetric modulated arc therapy differs from existing techniques like helical IMRT or intensity-modulated arc therapy (IMAT) because it delivers dose to the whole volume, rather than segment by segment. The treatment- planning algorithm ensures the treatment precision and helping to spare normal healthy tissue. ${ }^{7}$

Some of the studies were performed for the prostate cancer treatment; however, they included prostate only or prostate with seminal vesicles. ${ }^{8}$ The present study aims to expand such studies to identify the main and continual diversity between two techniques for prostate cancer cases that involve the seminal vesicles and pelvic lymph nodes. The dosimetric results and treatment delivery efficiency using the RapidArc technique were compared to those using the conventional static-gantry IMRT technique.

\section{Methods and Materials}

A cohort of 20 high-risk prostate cancers patients including prostate, seminal vesicles, and pelvic lymph nodes were selected for our study. Computed tomography (CT) images were acquired with an empty rectum and full bladder for all patients. The attending radiation oncologist manually segmented prostate, seminal vesicles, and nodes based on the CT images. The primary planning target volume $\left(\mathrm{PTV}_{\mathrm{P}}\right)$ was defined to include a $1.0 \mathrm{~cm}$ margin around the pelvic lymph nodes in all directions plus a $0.7 \mathrm{~cm}$ margin around prostate, and seminal vesicles in all direction except the posterior direction, where $0.5 \mathrm{~cm}$ margin was added. The boost planning target volume (PTV $)_{\mathrm{B}}$ was defined to include
$0.7 \mathrm{~cm}$ margin around the prostate and seminal vesicle in all direction except the posterior; where $0.5 \mathrm{~cm}$ margin was added.

Rectum, bladder, bowel, left and right femoral head were contoured as organ at risk (OARs) based on CT images. In all the patients, anterior-posterior (AP) and lateral separation were very close. The average AP diameters were $21 \mathrm{~cm}$ and the lateral diameters were $34 \mathrm{~cm}$. The average volume of $\mathrm{PTV}_{\mathrm{P}}$ and $\mathrm{PTV}_{\mathrm{B}}$ were $534.47 \mathrm{cc}$ (range, $860.10 \mathrm{cc}-360.00 \mathrm{cc}$ ) and $163.50 \mathrm{cc}$ (range, 181 cc-148 cc), respectively. The average volume of bladder, rectum, right femoral head, left femoral head and bowel were $223.51 \mathrm{cc}$ (range, $87.21 \mathrm{cc}-360.32 \mathrm{cc}$ ), $68.61 \mathrm{cc}$ (range, 42.91 cc-111.60 cc), 53.51 cc (range, 68.13 cc-40.31 cc), 53.47 cc (range, 37.90 cc-63.81 cc) and 1243.90 cc (range, 380 cc-1680 cc), respectively. For this study, we have followed Radiation Therapy Oncology Group (RTOG-0521) protocol. The total prescription dose was 75.6 Gy with a daily dose of $1.8 \mathrm{~Gy}$. The prescription dose of the primary plans was 45 Gy and an additional 30.6 Gy to boost plan. Two $\operatorname{arcs}\left(182^{\circ}\right.$ to $178^{\circ}$; $178^{\circ}$ to $182^{\circ}$ ) were used in both clock wise and anti-clock wise directions for all patients in RapidArc treatment planning. ${ }^{9}$ For the clockwise arc, the collimator was rotated $20^{\circ}$, whereas for the anti-clockwise arc, the collimated was rotated $340^{\circ}$ in order to reduce the effect due to inter-leaf leakage. Seven fixed fields with angulations of $0^{\circ}, 51^{\circ}, 102^{\circ}, 153^{\circ}$, $207^{\circ}, 208^{\circ}$, and $309^{\circ}$ with dynamic leaf were used for IMRT. All plans were generated using Eclipse treatment planning system (version 8.0.15) by a single user only and the volumetric dose optimization method followed the same systematic strategy regarding the objective and priorities. Dose grid sizes of $(2.5 \times 2.5 \times 2.5) \mathrm{mm}^{3}$ and the anisotropic analytical algorithm (AAA) were used in this study. The slice thickness of the CT images used for planning purpose was $3 \mathrm{~mm}$. Both IMRT \& RapidArc plans were developed for each patient using $6 \mathrm{MV}^{\mathbf{1 0}}$ photons with maximum dose rate of $600 \mathrm{MU} / \mathrm{min}$ in a Varian made Novalis Tx machine; having dynamic capability of 120 high definition Multi leave collimator (MLC). The optimization constraints ${ }^{9}$ for all plans are listed in Table 1. These constraints and weightings were set initially and then modified by either relaxing or tightening during the optimization process based on the real-time updated dose-volume histograms (DVHs) of structures.

Table 1: Dose-Volume constraints and relative weightings used for optimization of both FB and FFF RapidArc plans.

\begin{tabular}{llc}
\hline \hline PTVs and OARs & Dose-Volume constraints & Relative weighting \\
\hline PTV and PTV & $\mathrm{D}_{100} \geq 98 \% ; \mathrm{D}_{\max } \leq 102 \%$ & $120-130$ \\
Rectum and & $\mathrm{V}_{70 \%} \leq 30 \% ; \mathrm{V}_{50 \%} \leq 50 \% ; \mathrm{V}_{30 \%} \leq 70 \% ; \mathrm{D} 5-\mathrm{D} 10 \leq$ total of & $60-80$ \\
Bladder & $60-70 \mathrm{~Gy} ; \mathrm{D}_{\max } \leq 100 \%$ & \\
Bowel & $\mathrm{V}_{50 \%} \leq 30 \% ; \mathrm{V}_{30 \%} \leq 50 \% ; \mathrm{V}_{10 \%} \leq 70 \% ;$ & $50-60$ \\
& $\mathrm{D}_{\max } \leq$ total of 45Gy & $40-50$ \\
Femoral heads & $\mathrm{V}_{20 \%}-\mathrm{V}_{30 \%} \leq 30 \% ; \mathrm{D}_{\max } \leq$ total of 45Gy & \\
\hline
\end{tabular}




\subsection{Sigma index (S-index) and conformity index (CI)}

Treatment planning was done based on the radiotherapy objective of delivering a therapeutic dose to a well-defined target while minimizing dose to the surrounding normal tissue and critical organs. It requires optimization of conformity of the prescription dose to the planning target volume, dose homogeneity within the PTV, dose to the surrounding normal tissue and critical organs. The following parameters were evaluated for comparing cumulative DVH of the patient such as Sigma-index, conformity index, and mean doses to the bladder, rectum, bowel, right femoral head, and left femoral head.

A better representation of homogeneity could be defined in term of sigma index (S-index). ${ }^{11}$ This uses the differential DVH, unlike other indices, which is cumulative DVH. S-index is a measure of the standard deviation of the doses about the mean dose. The S-index is expressed as:

$$
\mathrm{S}-\text { index }=\mathrm{D}_{\mathrm{SD}}=\sum \sqrt{\left(\mathrm{D}_{\mathrm{i}}-\mathrm{D}_{\text {mean }}\right)} \times \frac{\mathrm{v}_{\mathrm{i}}}{\mathrm{V}}
$$

$\mathrm{D}_{\text {mean }}$ is the mean dose of the target (PTV in this study) curve. $D_{i}$ is dose to the $i^{\text {th }}$ bin having a volume $\mathrm{v}_{\mathrm{i}} . \mathrm{V}=$ total volume of the target.

The conformity index (CI) was defined to compare the treatment plans. The conformity index is defined as the ratio of the $95 \%$ isodose volume divided by the PTV that is enclosed by the $95 \%$ of the isodose line. From this definition, as the conformity index approaches 1 , the more conformal is the treatment plan.

\subsection{Dose distribution, PTV coverage and organs at risk}

In addition to providing information on the homogeneity of radiation doses, DVHs can be used to assess the target coverage index, defined as the percentage of the tumor volume that received the prescribed dose. Ideally, tumor DVH would be a step function, with $100 \%$ of the target receiving the exact prescribed dose. However, actual DVH curves are not step functions, because of constraints imposed by tumor volume and other OAR. Rules for the PTV was set such that the prescribed dose covered at least 95\% of the PTV (D95) and the PTV volume receiving $>107 \%$ of the prescription was limited to $2 \%$ in line with ICRU report 50 and ICRU report 62. To achieve this objective, a constraint for $\mathrm{D}_{100}$ was set to receive $\geq 98 \%$ of prescription and constraint for maximum dose $\left(D_{\max }\right)$ was set to receive $\leq 102 \%$ of the prescription in the optimization process for both plans. The volumes that received a minimum of $70 \mathrm{~Gy}, 60 \mathrm{~Gy}$, 40 Gy, (V70Gy, $V_{60 G y}, V_{40 G y}$ ) for rectum, $70 \mathrm{~Gy}, 60 \mathrm{~Gy}, 40$ Gy, (V70Gy, $V_{60 G y}, V_{40 G y}$ ) for bladder and $45 \mathrm{~Gy}, 15 \mathrm{~Gy}$
$\left(\mathrm{V}_{45 \mathrm{G} y}, \mathrm{~V}_{15 \mathrm{G} y}\right)$ for bowel, respectively, were selected to evaluate volumes of high dose. ${ }^{12-13}$

\subsection{Normal tissue integral dose and MU}

The integral dose (in unit of liter-Gy) was defined as the absorbed dose integrated over the voxels in the entire volume excluding the PTV. The integral dose (ID) (mean dose $\times$ tissue volume) received by normal tissue (NTID) 14 was calculated from dose-volume histograms. For comparison, the integral dose ratio was obtained by dividing the integral dose from IMRT plan by the integral dose from RapidArc plan. MU was analyzed for IMRT and RapidArc plans.

\subsection{Statistical analysis}

Statistical analysis was performed with Statistical Analysis Software (SAS), version 9.3. Mean values and standard deviation of the mean (SD) were collected. Relative dosimetric changes were compared applying the paired t-test. A two-sided $p$-value $\leq 0.05$ was considered statistically significant. Confidence intervals included $95 \%$ of the measured data.

\section{Results}

\subsection{Sigma index (S-index) and conformity index (CI)}

The S-index and conformity indices for all the prostate patients in the both IMRT and RapidArc plans with $6 \mathrm{MV}$ photons are analyzed. The relative efficacy (IMRT/ RapidArc) values for, S-index and conformity index are 0.9832, 1.010 for $\mathrm{PTV}_{\mathrm{P}}$ and 1.0, 1.005 for $\mathrm{PTV}_{\mathrm{B}}$ respectively. Table 2 shows S- index and conformity index of both IMRT and RapidArc Plans in all type of PTVs.

Table 2: S- index and Conformity index of both IMRT and RapidArc Plans in all type of PTVs.

\begin{tabular}{ccc}
\hline \hline Technique & SI & CI \\
\hline PTV IMRT & $2.35 \pm 0.581$ & $0.96 \pm 0.047$ \\
PTV RapidArc & $2.39 \pm 0.738$ & $0.95 \pm 0.059$ \\
PTV $_{\text {IMRT }}$ & $2.1 \pm 0.484$ & $0.979 \pm 0.015$ \\
PTV $_{B}$ RapidArc & $2.1 \pm 0.464$ & $0.975 \pm 0.014$ \\
\hline \hline
\end{tabular}

\subsection{Dose distribution, PTV coverage and organs at risk}

In this study, we analyzed a total of 80 prostate cancer treatment plans of 20 patients. The clinical dosimetric impact of IMRT, in comparison with RapidArc, was assessed. Transversal view of comparative dose distribution between IMRT plan (left side) and RapidArc plans for PTV $_{P}$ (right side) for prostate cancer with lymph nodes involved is shown in Figure 1. A typical DVHs for 6-MV plans (IMRT and RapidArc) are compared in Figure 2. 

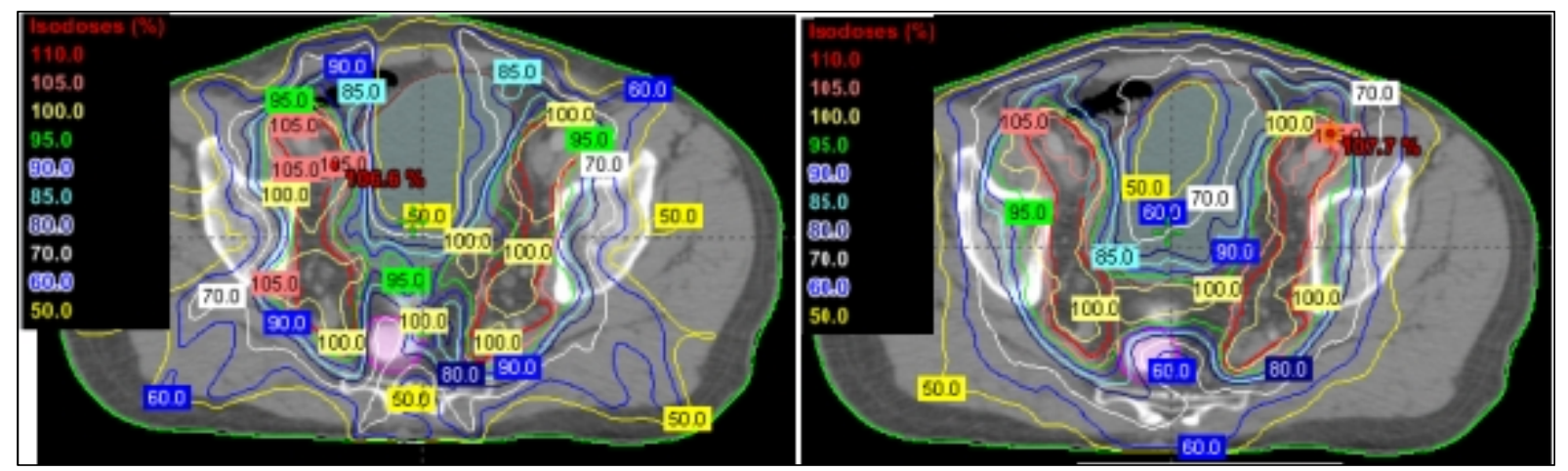

Figure 1: Transversal view of comparative dose distribution between IMRT plan (Left Side) and RapidArc plans for PTVP (Right side) for prostate cancer with lymph nodes involved.

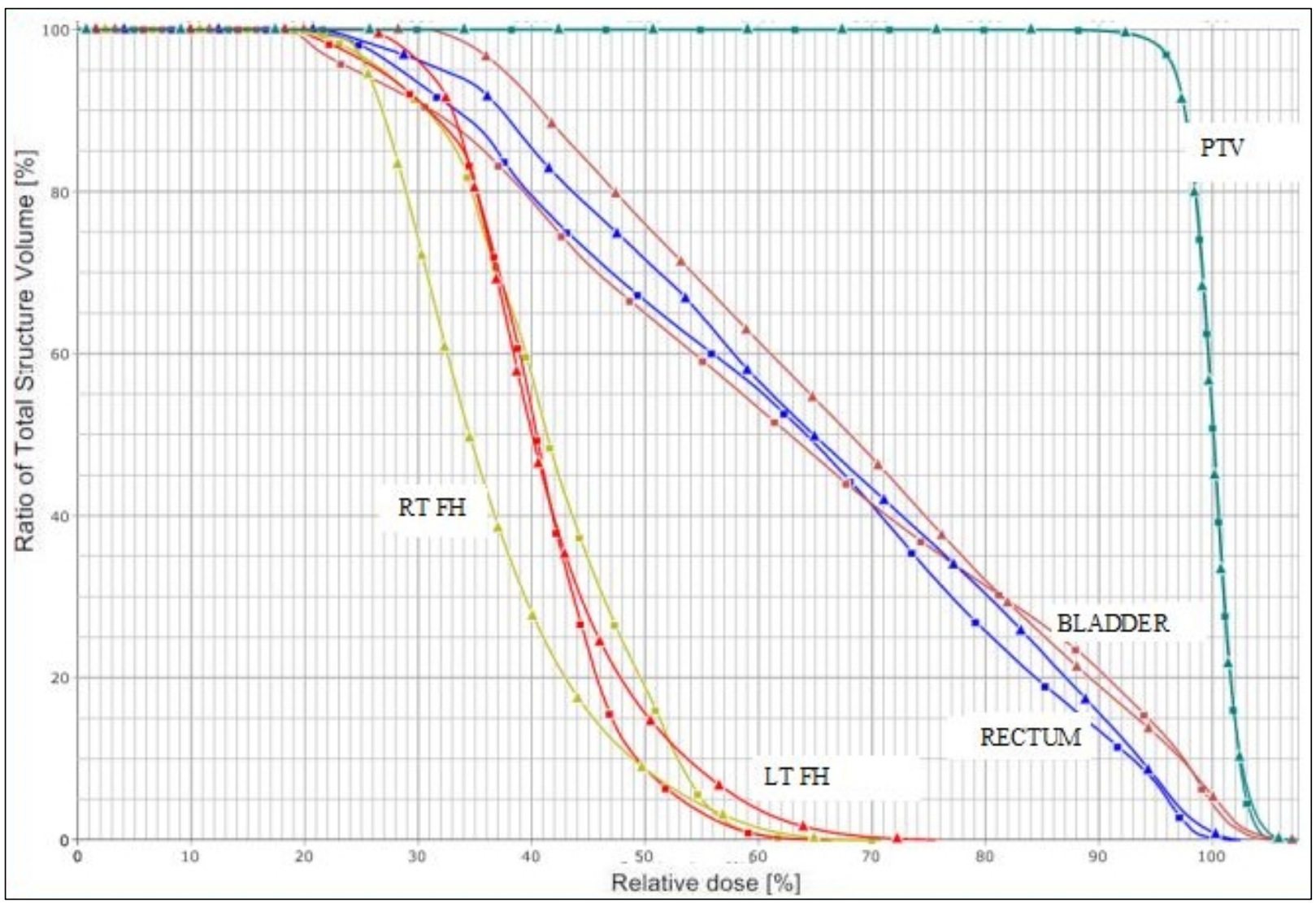

Figure 2: Comparison of the dose -volume histograms for IMRT (square) and (triangle) for RapidArc plan for the case shown in Figure 1.

Table 3: Mean dose statistics (with SD) of organ at risk for composite plan.

\begin{tabular}{cccc}
\hline \hline Structure & $\begin{array}{c}\text { Dose-volume } \\
\text { constraints }\end{array}$ & Volume achieved in $\%$ \\
\hline \multirow{3}{*}{ Rectum } & & IMRT & $\mathrm{RA}$ \\
& $\mathrm{V}_{70}<30 \%$ & $63.0 \pm 5.9 \%$ & $69.4 \pm 4.4 \%$ \\
& $\mathrm{~V}_{60}<50 \%$ & $50.0 \pm 4.1 \%$ & $57.4 \pm 3.0 \%$ \\
& $\mathrm{~V}_{40}<70 \%$ & $38.2 \pm 3.3 \%$ & $39.5 \pm 2.6 \%$ \\
Bladder & $\mathrm{V}_{70}<30 \%$ & $63.3 \pm 10.2 \%$ & $69.4 \pm 9.4 \%$ \\
& $\mathrm{~V}_{60}<50 \%$ & $45.0 \pm 7.4 \%$ & $49.5 \pm 7.7 \%$ \\
& $\mathrm{~V}_{40}<70 \%$ & $33.0 \pm 5.6 \%$ & $38.0 \pm 5.8 \%$ \\
Bowel & $\mathrm{V}_{45 \mathrm{~Gy}}<195 \mathrm{cc}$ & $4.49 \pm 1.08 \%$ & $5.00 \pm 1.09 \%$ \\
Rt. femoral head & $\mathrm{V}_{50 \mathrm{~Gy}}<5 \%$ & $0.0 \pm 0.0 \%$ & $0.0 \pm 0.0 \%$ \\
Lt. femoral head & $\mathrm{V}_{50 \mathrm{~Gy}}<5 \%$ & $0.0 \pm 0.0 \%$ & $0.0 \pm 0.0 \%$ \\
\hline \hline
\end{tabular}


For the rectum, there were dose differences between the two treatment modalities in the volume exposed to 70 Gy, 60 Gy, and 40 Gy are 10\%, 14.8\% and 3.4\%, respectively, which is statistically significant $(p<$ $0.0001)$. In case of bladder, there were dose differences for the volume exposed to $70 \mathrm{~Gy}, 60 \mathrm{~Gy}$ and $40 \mathrm{~Gy}$ are $9.63 \%, 10 \%$ and $15 \%$, respectively, which is statistically significant $(p<0.0001)$. For the bowel, there were no significant dose differences between two treatment modalities in the volume exposed to $45 \mathrm{~Gy}$ or $15 \mathrm{~Gy}$, and femoral heads were within the required constraints for all patients. Table 3 shows mean dose statistics with standard deviation of organ at risk for composite plan.

Table 4 shows the average maximum dose statistics (with SD) and average dosimetric indices with standard deviation of PTV and organ at risk for composite plan. There were significant differences between IMRT and RapidArc plans for the average doses delivered to the bladder and rectum. A significant increase in mean dose of $12 \%(p<0.0002,95 \% \mathrm{CI}=0.7714$ to 2.2312$)$ to the rectum and $15.4 \%(p<0.0001,95 \% \mathrm{CI}=4.294$ to 6.771$)$ for bladder was measured for RapidArc plans compared with IMRT plans. For the bowel, there was no significant dose difference between two treatment modalities in the average doses and femoral heads were within the required constraints for all patients. The average maximum dose of PTV is $106.7 \%$ and $105.7 \%$ of prescribe dose for both IMRT and RapidArc plans, respectively and different was significant $(p<0.0002$, $95 \% \mathrm{CI}=45.49$ to 127.5 ). No significant differences between two modalities were observed with respect to mean average maximum dose to bladder, rectum, femoral heads, and bowel.

\subsection{Normal tissue integral dose (NTID) and MU}

NTID was used as an index to compare techniques for radiation induced second cancers. The NTID (mean \pm SD) was $182.07 \pm 46.07$ liter-Gy for IMRT plan and $183.65 \pm 47.26$ liter-Gy for RapidArc plans. RapidArc plans produced $0.87 \%$ ( $p<0.044,95 \% \mathrm{CI}=0.045$ to 3.113) more dose compared with the IMRT beam, and this difference is not significant. Comparison of the integral dose for IMRT and in RapidArc composite plans is shown in Figure 3. We found that MU (mean \pm SD) was $1542 \pm 239$ for IMRT plans and $618 \pm 71$ for RapidArc plans in PTV. MU (mean \pm SD) was $1041 \pm 195$ for IMRT plans and $605 \pm 78$ for RapidArc plans in PTV Compared with RapidArc plans, the MU significantly increased by 2.50 times $(p<0.0001,95 \% \mathrm{CI}=368.7 .49$ to 502.3) in $\mathrm{PTV}_{\mathrm{P}}$ and 1.72 times $(p<0.0001,95 \% \mathrm{CI}=$ 846.4 to 998.2) in PTV $_{B}$ for IMRT plans shown in Figures 4-5. The beam-on time of PTVp was $4.2 \mathrm{~min}$ to $4.3 \mathrm{~min}$ for IMRT and $2.1 \mathrm{~min}$ to $2.3 \mathrm{~min}$ for RapidArc; whereas for PTV $\mathrm{B}_{\mathrm{B}}$, it was $2.5 \mathrm{~min}$ to $2.6 \mathrm{~min}$ for IMRT and $1.0 \mathrm{~min}$ to 2.3 min for RapidArc. Treatment time of PTVp was 8.1 min to $8.2 \mathrm{~min}$ for IMRT and $3.1 \mathrm{~min}$ to $3.2 \mathrm{~min}$ for RapidArc; whereas for PTV $_{B}$, it was $5.1 \mathrm{~min}$ to $5.2 \mathrm{~min}$ for IMRT and $3.1 \mathrm{~min}$ to $3.2 \mathrm{~min}$ for RapidArc. The treatment time of RapidArc was 1.5 times shorter than IMRT for PTVp but approximately same in PTV $_{\text {В }}$ plans.

Table 4: Average maximum dose statistics (with SD) and average dosimetric indices (with SD) of PTV and organ at risk for composite plan.

\begin{tabular}{ccccc}
\hline \hline Structure & \multicolumn{2}{c}{ Mean dose in Gy } & \multicolumn{2}{c}{ Maximum dose in Gy } \\
\hline & IMRT & RA & IMRT & RA \\
PTV & $76.50 \pm 0.82$ & $76.50 \pm 0.72$ & $80.89 \pm 1.31$ & $80.13 \pm 0.93$ \\
Rectum & $37.9 \pm 4.75$ & $42.7 \pm 4.59$ & $76.44 \pm .68$ & $77.77 \pm .75$ \\
Bladder & $35.7 \pm 11.86$ & $41.2 \pm 12.0$ & $79.46 \pm 0.56$ & $78.92 \pm 0.66$ \\
Bowel & $23.0 \pm 2.89$ & $23.08 \pm 2.84$ & $45.6 \pm 24.2$ & $45.7 \pm 23.6$ \\
RT FH & $24.5 \pm 1.88$ & $25.3 \pm 1.82$ & $47.47 \pm 4.44$ & $49.35 \pm 5.13$ \\
LT FH & $24.4 \pm 1.85$ & $25.2 \pm 1.97$ & $47.86 \pm 4.60$ & $50.51 \pm 6.02$ \\
\hline \hline
\end{tabular}

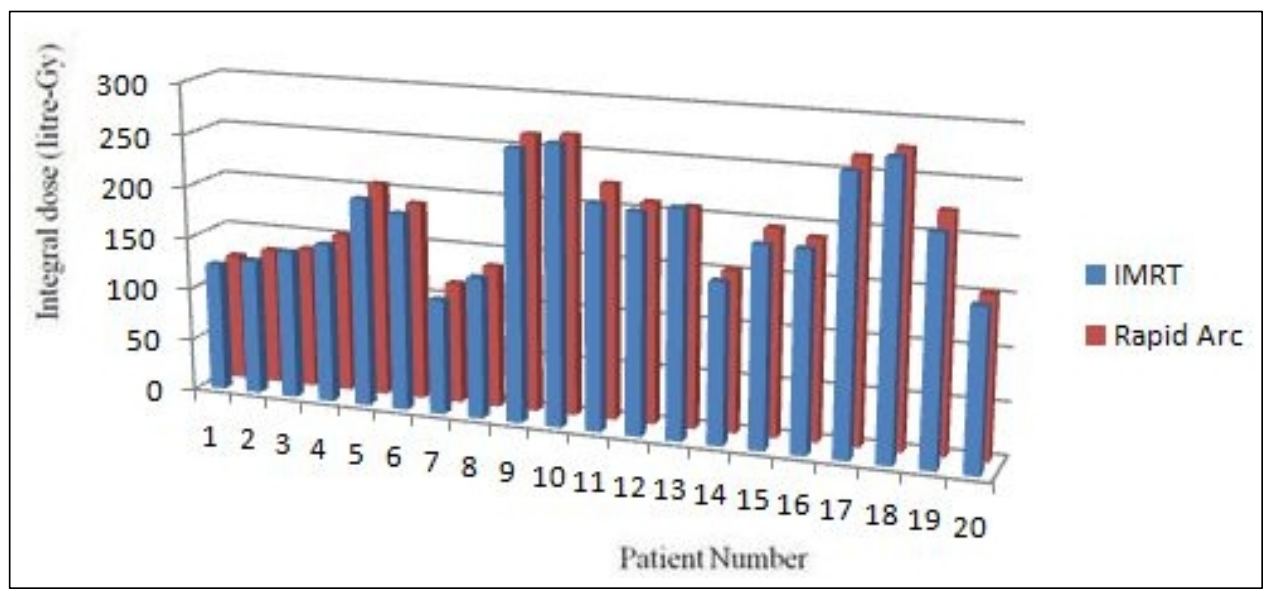

Figure 3: Comparison of the integral dose for IMRT and RapidArc composite plans. 


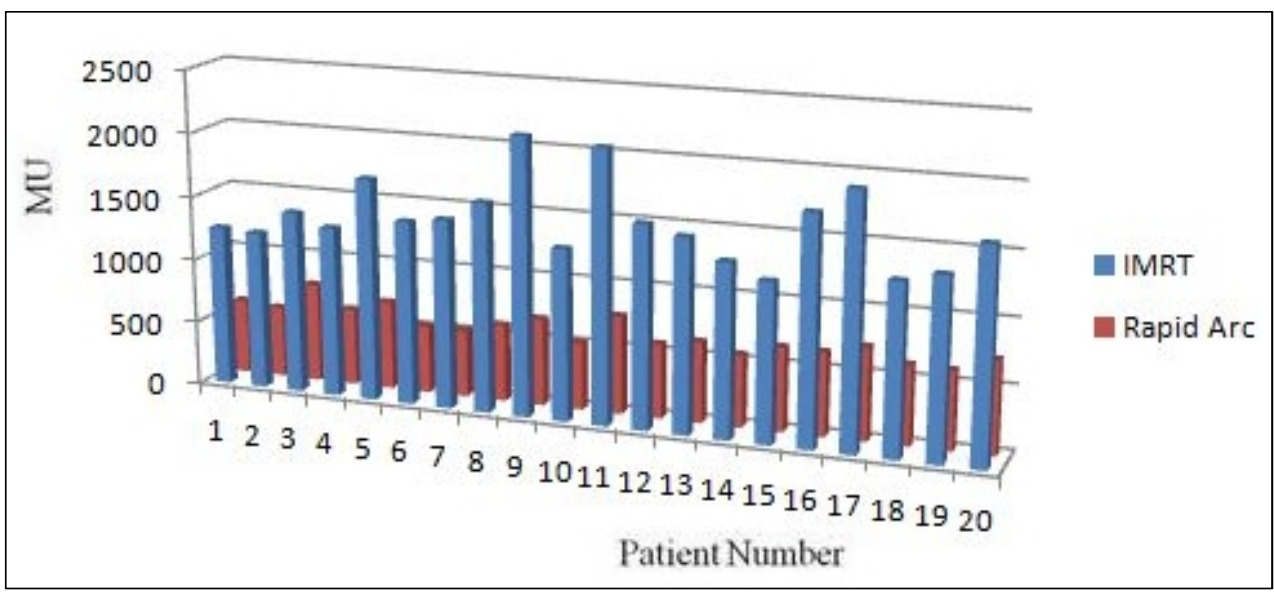

Figure 4: Comparison of the MUs for IMRT and RapidArc plans for PTVP (primary planning treatment volume).

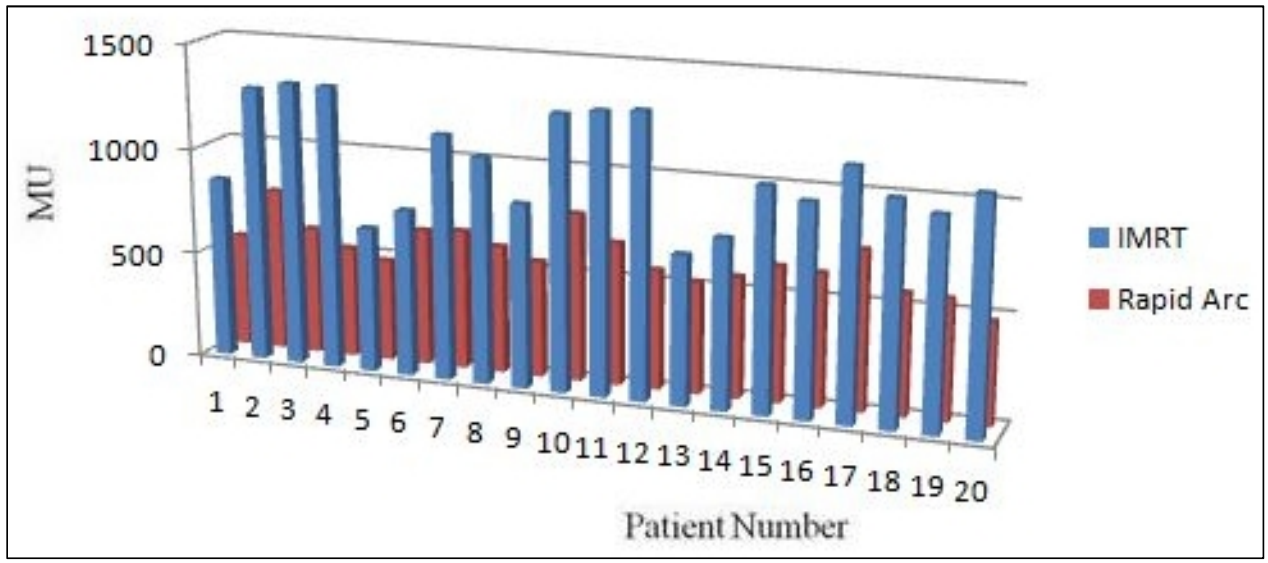

Figure 5: Comparison of the MUs for IMRT and RapidArc plans for PTVB (Boost planning treatment volume).

\section{Discussion}

It clearly represents that there is no significant difference of homogeneous index for both the PTVs in IMRT and RapidArc plans. From our data between IMRT and RapidArc plans, IMRT plans are showing lower S-indices for both $\mathrm{PTV}_{\mathrm{P}}$ and $\mathrm{PTV}_{\mathrm{B}}$ plans indicating improved dose homogeneity compared to RapidArc plans. However, target inhomogenity (defined as Dmax-Dmin) was increased by $12.3 \%$ ( $p<0.0001,95 \%$ $\mathrm{CI}=2.256$ to 3.677 ) for IMRT compare with RapidArc composite plans. For IMRT plans, target inhomogenity is significantly increased for $\mathrm{PTV}_{\mathrm{p}}$ compared to RapidArc plans because of lymph nodes involvement with PTV. However, in $\mathrm{PTV}_{\mathrm{B}}$, inhomogenity is comparable for both plans.

Miften et al. ${ }^{15}$ have demonstrated the use of target conformity index (TCI) and normal tissue-sparing index (NTSI) to assist in the process of judging the merit of a clinical treatment plan. However, in this work, the widely accepted conformity index was used to evaluate the conformity of the treatment plans (Table 2) for all the patients using both techniques. The mean conformity index was 0.96 and 0.95 for $\mathrm{PTV}_{\mathrm{P}}$ and 0.979 and 0.975 for $\mathrm{PTV}_{\mathrm{B}}$ in both IMRT and RapidArc plans, respectively. These small differences indicate that these plans are nearly identical in their conformity of dose to the target. However, there is difference observed in between PTVs. For PTV $\mathrm{P}_{\mathrm{P}}$ conformity index is lower values than $\mathrm{PTV}_{\mathrm{B}}$ plans due to involvement of lymph nodes. Sua et al.16 reported similar results in their planning study for PTVs involved with pelvic lymph nodes.

Rectum and bladder are showing high dose for RapidArc plans comparable to IMRT plans. This dose variation was significantly increased in PTVp compared to $\mathrm{PTV}_{\mathrm{B}}$. Sua etal. ${ }^{16}$ reported that IMRT plans with PTV $_{P}$ showed better sparing of bladder, rectum, and small bowel than 2 arcs plans. Kjaer-kristoffersen et al. ${ }^{17}$ noted larger OAR doses with RapidArc than IMRT plans while prostate and seminal vesicles were considered as PTVs.

Because radiation could induce second malignancies ${ }^{\mathbf{1 8}}$, ${ }^{19}$, integral dose was used as an index to evaluate treatment plans. In our study, integral dose index (IMRT/RapidArc) is 0.99 , which is very negligible. Sua et al. ${ }^{16}$ reported that $7 \%$ to $8 \%$ greater integral dose in RapidArc than in IMRT which is not confirmed in our study. Palmer et al. ${ }^{20}$ noted larger integral doses with IMRT than with IMAT based on MU values for prostate 
cancer which is conflict with our findings. Integral dose is related not only to MUs but also to other complicated factors. The combination of MUs, corresponding aperture sizes and shapes, target volumes and shapes contributes to integral dose. ${ }^{16}$ In the primary plans, MUs was approximately $60 \%$ greater than in RapidArc plans. In boost plans, the average values of total MUs in IMRT plans was approximately $42 \%$ greater than in RapidArc plans.

The IMRT planning was performed in Eclipse treatment planning system using Novalix Tx Linac and 120HD MLC, which have a limit that MLC cannot travel beyond $14 \mathrm{~cm}$ during beam delivery. To overcome this limit, a large IMRT beam is split into two or three subfields, which are planned and calculated as one beam but delivered as two or three separate beams. ${ }^{16}$ Without this limitation, IMRT delivery time could be reduced by approximately $1.2 \mathrm{~min}$ for a prostate cases that include the lymph nodes. The mean delivery time of IMRT was longer owing to the larger number of beams, the dead time in the gantry rotation from field to field and the larger number of MUs delivered. The shorter treatment time of RapidArc is beneficial to the patient, as it would help reduce the time the patients required to maintain a full bladder status. Shorter treatment time does not only reduce the probability of the intra-fractional motion of patients, but it can also decrease the impact of internal organ motion on the treatment delivery. ${ }^{21}$ The shorter treatment time of RapidArc would be welcomed by busy centers and shorten the patient waiting list.

Although our study focused on photon therapy, proton therapy is also another option to treat the prostate cancer in external beam radiotherapy. ${ }^{22,23}$ Proton therapy can produce excellent dose distribution because protons have finite range and sharp distal fall-off at the end of proton beam path. Vargas et al. ${ }^{24}$ and Rana et al. ${ }^{25}$ demonstrated that proton therapy is better at sparing rectum and bladder when compare to the photon therapy.

\section{Conclusion}

In this study, the clinical dosimetric impact of IMRT, in comparison with RapidArc, was assessed for prostate cancer patients. For complicated and large PTVs that included prostate, seminal vesicles and lymph nodes, conventional IMRT spared the bladder, rectum doses better than did RapidArc. For simple and small PTVs that included prostate and seminal vesicles, RapidArc plans were comparable to those achieved with conventional IMRT plans. The integral dose was more in RapidArc but not significant. The treatment delivery efficiency improved with RapidArc plans. RapidArc plans should be compared with IMRT plans for individual cases to measure gains and losses before selecting one over the other. Clinical studies are required to evaluate its clinical benefits.

\section{Conflict of interest}

The authors declare that they have no conflicts of interest. The authors alone are responsible for the content and writing of the paper.

\section{References}

1. Ferlay J, Soerjomataram I, Ervik M, et al, GLOBOCAN 2012 v1.0, Cancer Incidence and Mortality Worldwide: IARC Cancer Base No. 11, Lyon, France: International Agency for Research on Cancer. Available from http://globocan.iarc.fr. 2013.

2. Hall EJ. Intensity modulated radiation therapy, protons, and risk of second cancers. Int J Radiat Oncol Bio Phys. 2006; 65:1-7.

3. Nguyen PL, Gu X, Lipsitz SR, et al. Cost implications of the rapid adoption of newer technologies for treating prostate cancer. J Clin Oncol. 2011; 29:1517-24.

4. Rukstalis DB. Treatment options after failure of radiation therapy-a review. Rev Urol. 2002; 4 Suppl 2:S12-7.

5. Heidenreich A, Bolla M, Joniau S, et al. Guidelines on prostate cancer. European association of urology, 2010.

6. Otto K. Volumetric modulated arc therapy: IMRT in a single gantry arc. Med Phys. 2008; 35:310-7.

7. Ojala J. The accuracy of the Acuros XB algorithm in external beam radiotherapy - a comprehensive review. Int J Cancer Ther Oncol 2014; 2:020417.

8. Rana S, Rogers K, Lee T, et al. Dosimetric impact of Acuros XB dose calculation algorithm in prostate cancer treatment using RapidArc. J Cancer Res Ther. 2013; 9:430-5.

9. Rout BK, Muralidhar KR, Ali M, et al. Dosimetric study of RapidArc plans with flattened beam (FB) and flattening filter-free (FFF) beam for localized prostate cancer based on physical indices. Int J Cancer Ther Oncol. 2014; 2:02046.

10. Sun M, Ma L. Treatment exceptionally large prostate cancer patients with low-energy intensity-modulated photons. J Appl Clin Med Phys. 2006; 7:43-9.

11. Yoon M, Park SY, Shin D, et al. A new homogeneity index based on statistical analysis of the dose-volume histogram. J Appl Clin Med Phys. 2007; 8:9-17.

12. Marks LB, Yorke ED, Jackson A, et al. Use of normal tissue complication probability models in the clinic. Int J Radiat Oncol Biol Phys. 2010; 76:S10-9.

13. Michalski JM, Purdy JA, Winter K, et al. Preliminary report of toxicity following 3D radiation therapy for prostate cancer on 3DOG/RTOG 9406. Int J Radiat Oncol Bio Phys. 2000; 46:391-402. 
14. Followill D, Geis P, Boyer A. Estimates of whole-body dose equivalent produced by beam intensity modulated conformal therapy. Int J RadiatOncol Biol Phys. 1997; 38:667-72.

15. Miften MM, Das SK, Su M, Marks LB. A dose-volume based tool for evaluating and ranking IMRT plans. J Appl clin Med Phys. 2004; 5:1-14.

16. Yoo S, Wu QJ, Lee WR, Yin FF. Radiotherapy treatment plans with RapidArc for prostate cancer involving seminal vesicles and lymph nodes. Int J Radiat Oncol Biol Phys. 2010; 76:935-42.

17. Kjaer-Kristoffersen F, Ohlhues L, Medin J, Korreman S. RapidArc volumetric modulation therapy planning for prostate cancer patients. Acta Oncol. 2009; 48:227-32.

18. Brenner DJ, Curtis RE, Hall EJ, Ron E. Second malignancies in prostate carcinoma patients after radiotherapy compared with surgery. Cancer. 2000;88:398-406.

19. Hall EJ, Wuu CS. Radiation-induced second cancers: The impact of 3D-CRT and IMRT. Int J Radiat Oncol Biol Phys. 2003; 56:83-8.

20. Palma D, Vollans E, James K, et al. Volumetric modulated arc therapy for delivery of prostate radiotherapy: Comparision with intensity modulated radiotherapy and three dimensional conformal radiotherapy. Int J Radiant Oncol Biol Phys. 2008;72:996-1001.

21. Isa M, Rehman J, Afzal M, Chow JC. Dosimetric dependence on the collimator angle in prostate volumetric modulated arc therapy. Int J Cancer Ther Oncol 2014; 2:020419.

22. Tesfamicael BY, Avery S, Gueye P, et al. Scintillating fiber based in-vivo dose monitoring system to the rectum in proton therapy of prostate cancer: A Geant4 Monte Carlo simulation. Int J Cancer Ther Oncol. 2014; 2:02024.

23. Rana $\mathrm{S}$, Larson G, Vargas C, et al. Intensity modulated proton therapy versus uniform scanning proton therapy: Treatment planning study of the prostate cancer in patients with a unilateral metallic hip prosthesis. Jour Proton Ther. 2015; 1:113.

24. Vargas C, Fryer A, Mahajan C, et al. Dose-volume comparison of proton therapy and intensity-modulated radiotherapy for prostate cancer. Int J Radiat Oncol Biol phys. 2008; 70:744-51.

25. Rana S, Cheng C, Zheng Y, et al. Proton therapy vs. VMAT for prostate cancer: a treatment planning study. Int J Particle Ther. 2014;1:2233. 